\title{
IMPACT OF CORPORATE SUSTAINABILITY REPORTING PRACTICE ON CORPORATE PERFORMANCE: A REVIEW OF LITERATURE
}

\author{
Nusirat Ojuolape Gold ${ }^{1,2, *}$, Fauziah Md. Taib1 \\ ${ }^{1}$ School of Management, Universiti Sains Malaysia, Penang, Malaysia \\ ${ }^{2}$ Department of Accounting and Finance, Kwara State University, Malete, Nigeria
}

ABSTRACT - Stakeholder pressure on corporations to adopt sustainable practice has been a subject steering constant argument in recent times. The reason could be due to the awareness of the environmental impact and concern in preserving the planet. This study, through a literature review of prior studies, examined the impact of corporate sustainability reporting practice on corporate performance. Numerous researchers investigated this relationship in the past, but there is still a lack of consensus with regards to outcomes. Findings have been inconsistent and contradictory, varying from positive to a negative relationship, to statistically insignificant or mixed outcomes dependent on several factors such as cost exceeding benefits, shareholders perceiving sustainability initiative as a cost object, investors not valuing disclosure, firms using disclosure as legitimization tool for prestige, weak legislation, proxies used for measurement tool, country/ region of study, methodology, the period covered, industry sector covered and/or sample size. Our study reviewed 35 works of literature and found 13 studies with positive outcomes, 8 with significant negative outcomes, 5 with no significant relationship and 9 with mixed result. Overall, we concluded that it pays for corporations to adopt sustainable business practices as the benefit of adoption will span across the long term. Not denying the fact that companies would incur some huge costs in the short run at the time of investment, but the long-run benefits would far outweigh whatever cost they might have incurred. Hence, corporations are advised to start incorporating the sustainable practice into the management process and subsequently report on them to avoid legitimacy cost and to gain long-run competitive advantage. Future studies may conduct a systematic review to disaggregate the approaches, so as to examine the different dimensions of sustainability practice and provide a more concise and clear result.
ARTICLE HISTORY

Received: $16-9-2020$

Accepted: $1-12-2020$

\section{KEYWORDS}

Sustainability Reporting

Practice,

Corporate Performance,

Sustainability Disclosure,

Global Reporting Initiative,

Triple Bottom Line

\section{INTRODUCTION}

Stakeholder pressure on corporations to adopt sustainable practices has been a subject steering constant argument recently. Reasons could be due to the awareness of the environmental impact and concern in preserving the planet. Moreover, the rising climatic temperature is driven by the high concentration of greenhouse gases (GHGs) in the atmosphere which has caused an outbreak of diseases, loss of wildlife and aquatic animals, and loss of lives and property among others. In a bid to reverse this trend, stakeholders demand corporate reporting on sustainability, since corporate transparency and accountability are assumed key (Chithambo, Tingbani, Agyapong, Gyapong, \& Damoah, 2020). Given the pressure, corporations began publishing the impact of their activities (positive and negative impact) on the environment in a sustainability report as a voluntary disclosure or as mandatorily required under different jurisdictions.

Furthermore, sustainability reporting forms an integral part of Integrated Reporting because it combines financial and non-financial parameters. Using the definition given in the Brundtland Report of 1987, sustainability is said to be a development that meets the needs of the present without compromising the ability of future generations to meet their own needs (World Commission on Environment and Development, 1987). This definition is the most widely accepted definition of sustainability since it captures both current and future generation needs. However, corporate sustainability has grown from a mere emphasis on promoting environmental, social, and governance (ESG) performance to concern that can derive revenue growth and high quality financial performance as businesses and global investors began applying sustainability performance information and look beyond a company's financials in making business and investment decisions (Rezaee, 2016).

Meanwhile, with the upsurge pressure on companies to embrace this form of reporting, and to ensure uniform disclosure by them, several frameworks and standards were developed. For instance, Global Reporting Initiative (GRI), Dow Jones Sustainability Index (DJSI), Carbon Disclosure Project (CDP), Global Initiative for Sustainability Ratings (GISR), International Integrated Reporting Council (IIRC), Sustainability Accounting Standards Board (SASB), etc. The GRI remains the most popular among them (KPMG, 2017) providing corporations and businesses with strategic ways to analytically assess, measure, and communicate their economic, social, and environmental performance. Hence, if 
companies were to adopt sustainable business practices, it is believed to pave way for corporate goal of value creation which will be achievable when management considers not just shareholders interest (i.e. profit maximization) but also considers all stakeholders' interest by integrating non-financial dimensions of sustainability into corporate strategies and business processes.

Another perspective to explain sustainability reporting as given by Global Reporting Initiative (2016) is an organization's practice of reporting publicly on its economic, environmental, and/or social impacts; and the organization's positive or negative contributions towards achieving the goal of sustainable development. This view conforms with the triple bottom-line approach of people, profit, and planet that business activities can deliver financial, social, and environmental benefits simultaneously (Henriques \& Richardson, 2004). To this end, GRI requires corporations to set an equilibrium among economic, environmental, and social needs so as not to jeopardize future development. Correspondingly, sustainability reporting is generally believed to lay foundation for preserving and enhancing firm value through strategic benefits such as improved stakeholder engagement or relations, better customer access, customer loyalty, new products creation, identification of new markets, and operational improvements (Furlan Alves, Lopes de Sousa Jabbour, \& Barberio Mariano, 2019), improved reputation (Hoejmose, Roehrich, \& Grosvold, 2014), gaining employee loyalty (Kwaghfan, 2015), risk avoidance, gain access to financial capital (Schmidt, Foerstl, \& Schaltenbrand, 2017), cost savings, productivity, etc. (Aggarwal, 2013).

As the calls to adopt sustainable practice are still predominant, stakeholders especially investors are now looking into investing in firms that are deemed socially responsible, although, the number of companies adopting and reporting on sustainability aspects is on the rise. However, according to the international survey on corporate responsibility reporting carried out by KPMG in 2017, only $28 \%$ of companies worldwide acknowledge in their annual reports that climate change poses a financial risk (KPMG, 2017), implying the road ahead is still very far. Numerous researchers and academicians investigated the relationship between sustainability reporting and corporate performance using different parameters (Borges Junior, 2019; Buallay, 2019; Zhao et al., 2018), but findings failed to reach a consensus. Results have been equivocal as researchers found either positive (Ameer \& Othman, 2012; Borges Junior, 2019; Buallay, 2019), or contrarily, others found negative (Dinçer \& Altınay, 2020; Fatemi, Glaum, \& Kaiser, 2018; Rajesh \& Rajendran, 2020), mixed (Akbulut \& Kaya, 2019; Sampong, Song, Boahene, \& Wadie, 2018) or no significant association (Gunarsih \& Ismawati, 2018; Yilmaz, Aksoy, \& Tatoglu, 2020).

On the basis of an extensive review of literature, our study objective is to provide a nomenclature of prior existing studies to enable better insight in understanding sustainability reporting by corporations and provide useful guidance for future research in the area. Specifically, we gave explanation to the concept, provide theoretical explanations on the linkage between sustainability reporting and corporate performances, and review findings of extant literature to proffer robustness for the study results, conclusion, and future investigations. The study is timely as authorities, market regulators, and operators around the globe are effortlessly initiating sustainability policies, regulations, and encouraging good corporate governance and transparency among companies. For instance, the Nigerian Stock Exchange (NSE) is set to implement sustainability reporting practices for listed firms as of September 2020 (NSE, 2018). The study will also avail researchers knowledge on the findings of prior literature from countries around the globe that had already implement sustainability reporting and serve as a good ground for companies to embrace full-fledged sustainability practice encompassing mere economic, social and environmental reporting, while also enlightening organizations on the need to strive for interest of all stakeholders beyond the ordinary goal of increased profitability for the shareholders.

The next aspect after this section is the conceptual underpinning, followed by theoretical explanations and a summary of empirical findings from extant studies. Subsequently, the following sections tell about the methodology adopted by the study and the final section will be about the conclusion, contribution to knowledge, and recommendations for future research.

\section{LITERATURE REVIEW}

\section{Conceptual Underpinning}

\section{Sustainability Reporting Practice}

The concept of Sustainability Reporting practice evolved since the 80 s when the first environmental report was published. However, the concept has gained more attention from regulatory bodies, market participants, academicians, and corporations (Shad, Lai, Fatt, Klemeš, \& Bokhari, 2019) more recently. Oftentimes, the concept is usually coined interchangeably as Corporate Responsibility Reporting (CRR), Environmental Reporting (ER), Environmental, Social and Governance (ESG) reporting, Sustainability reporting, Corporate ESG Reporting, Integrated Reporting or Triple Bottom Line of people, profit and planet (Elkington, 1999; Ioannou \& Serafeim, 2017; Ng \& Rezaee, 2012; Wei, 2020).

Sustainability reporting refers to the ability of an organization to utilize the limited resources at its disposal effectively and efficiently over time by adopting strategies to minimize waste and uphold best corporate practices. Sustainability Reporting comprises of all the three dimensions of economic, environmental, and social sustainability while the Sustainability Practice span beyond mere reporting on the three dimensions (Rajesh, 2020). Hence, it provides a framework to create value above achieving sufficient profits but also satisfying the diverse needs of different stakeholder groups (López, Garcia, \& Rodriguez, 2007).

Since the concept comprises of three aspects of economic, social, and governance (ESG), it is vital to explain each of the dimensions. The economic dimension according to GRI (2002) is concerned with an organization's impacts on the 
economic circumstances of its stakeholders and the economic systems at local, national, and global levels. Shad et al. (2019) refer to the economic dimension as covering economic prosperity, profit-making, attaining competitive advantage, and sustaining the overall economic value of the corporation. The environmental dimension relates to environmental quality focusing on climate change impact, global warming, pollution, and depletion of ozone layer; an aspect explaining how organization's activities impact both living and non-living natural ecosystems. According to Delai and Takahashi (2013), the environmental dimension goes beyond the ecosystem's wellbeing because the ecosystem maintains diversity and quality, hence, its ability to support all life, and the potential to adapt to change to provide future options. Lastly, it is the social dimension which centers on how organizations impact the social system within which the company operates. This form of impact relates to social progress such as health and safety, community well-being, employment opportunities, charity, and organizational behavior (Aras, Tezcan, \& Kutlu Furtuna, 2018). In some cases, social indicators may influence the organization's intangible assets, such as its reputation or brand name.

Certainly, when corporations adopt sustainability reporting practices, they must be able to achieve equilibrium between firm business risk and meeting stakeholder expectations. Likewise, if they seek business performance in a socially responsible manner, the organization needs to connect sustainability management framework that will assist in predicting corporate performance (Maletič, Maletič, \& Gomišček, 2018 as cited by Shad et al., 2019) and that which will help in the transformation of a set of technical concepts into political and business policies and practices having a direct linkage to organizational performance (Shad et al., 2019).

\section{Corporate Performance}

Corporate performance in this instance may be interpreted as the ability of a firm to utilize resources at its disposal judiciously and implement activities effectively and efficiently better than its rivals. Corporate performance, according to Clarkson (1995), is concerned with measuring stakeholders' satisfaction by evaluating data concerning the actions and records of the company about the management of particular stakeholder issues and the levels of responsibility that the company has assumed. Thus, this author refers performance as "doing less or more, than is required" either as stipulated by legislation or specifically by the company's code of responsibilities and obligations. Although wide debate suggests no concise definition exists for corporate performance, yet, performance can be used as a basis to explain the management of a corporation's relationships with its stakeholders using concepts concerning corporate social responsibilities and responsiveness. Studies have measured corporate performance employing either a market-based approach or accountingbased metrics. The most commonly used metrics are return on asset (ROA), return on equity (ROE), Tobin's Q, profit margin, sales growth, cash flow, and stock prices, etc.

\section{Theoretical Explanations}

\section{Stakeholders Theory}

Stakeholders are persons or groups with legitimate interests in procedural and substantive aspects of corporate activity. Stakeholder theory anchors on the belief that other groups exist to whom the organization owes responsibility aside from equity shareholders. This refers to people or groups with stakes in the organization whose actions and inactions can influence or be influenced by the firms' decisions (Freeman, 1984). Stakeholders comprise shareholders, employees, customers, suppliers, lenders, regulatory authorities, and other members of the society without whose support the company would not function well or cease to exist (Freeman, Harrison, Wicks, Parmar, \& De Colle, 2010). Proponent of this theory, however, Donaldson and Preston (1995), and Freeman and David (1983) argued that stakeholder analysis continually forms an integral part of sustainability reporting practice and corporate planning process. They also opine corporations that practice stakeholder management will be relatively successful in terms of conventional performance such as profitability, stability, and growth. Hence, if a company fails to uphold the interest of any stakeholder group, it may jeopardize the corporate reputation and subsequently affect company performance.

\section{Legitimacy Theory}

Legitimacy, according to Suchman (1995), refers to "a generalized perception or assumption that the actions of an entity are desirable, proper, or appropriate within some socially constructed system of norms, values, beliefs, and definitions". Legitimacy is assumed generalized because it embodies an umbrella evaluation that transcends specific adverse events and occurrences from past events (Suchman, 1995). The theory relies on the firm belief that it is very vital for organizations to meet societal values, norms, and expectations to ensure their continuous existence, growth, and longterm survival. Proponents of the theory, Brown and Deegan (1998), Deegan (2002), and Patten (1991) posited that sustainability reporting practice will assist to reduce regulatory risk and adverse reactions of stakeholders, thereby strengthening organizational license to operate. Whereas, legitimacy is socially constructed because it reflects a congruence between the behaviors of the legal entity and the shared beliefs of some social group. Besides, legitimacy theory is a reflection of a social contract, which implies the firm's survival is dependent on its extent to operate within the bounds and norms of society (Brown \& Deegan, 1998).

\section{Empirical studies}

Extant studies have investigated the relationship between sustainability reporting and corporate performance in the past with emphasis laid on sustainability reporting, corporate social performance, and corporate financial performance. Margolis and Walsh (2003) assessed how far organization theory and empirical research responded to the tension over 
corporate involvement in wider social life. To explore the link in this relationship, they evaluated 127 published studies between 1972 and 2002 out of which, 4 studies found a bi-directional relationship, 54 studies from about 109 studies that treated sustainability performance as an independent variable reveal a positive relationship, 7 found negative relationship, 28 reveal no significant relationship while 20 had mixed findings. 16 studies out of those that made corporate sustainability a dependent variable reported a positive relationship. Al-Tuwaijri, Christensen, and Hughes (2004) conducted simultaneous equations models to provide an integrated analysis of the interrelations among environmental disclosure, environmental performance, and economic performance following the argument that management's overall strategy will affect each aspect of a corporation responsibilities. They obtained results that suggest good environmental performance is significantly associated with good economic performance, and with extensive quantifiable environmental disclosures of specific pollution measures and occurrences.

Muhmad and Muhamad (2020) in their review of sustainable business practices and financial performance during preand post-SDG adoption periods identified trends and issues highlighted in previous studies concerning the relationship between sustainability practice and financial performance. The author adopted content analysis to examine 56 studies indexed in the web of science (WoS) and Scopus with the majority from the developed countries and found that about $96 \%$ of the publications reported a positive relationship between sustainability practices and the financial performance of companies. From the review of extant literature, some authors proxied performance with return on assets (ROA), return on equity (ROE), return on sales (ROS), profit before taxation (PBT), and cash flow from operations (CFO) all of which are accounting-based measures while others use market-based measures such as Tobin's Q, stock returns, share prices, market value added (MVA), capital asset pricing model (CAPM), etc.

However, despite extensive empirical investigations, study findings have been equivocal as they fail to provide a clear and precise relationship between sustainability reporting and corporate performance. One plausible identified reason for the contradictory results is measurement issues pertaining to both concepts of interest.

\section{METHODOLOGY}

We based our review article on a qualitative and descriptive research approach that mainly centers on examining, analyzing, and summarizing the findings and limitations of prior relevant studies and other research sources relating to the research objectives. Borges Junior (2019) defined descriptive research approach as one whose focal purpose is to describe the characteristics of a phenomenon or population and establish relationships between variables. For the purpose of this review study, we collected our research articles from several databases including Web of Science (WoS), Taylor \& Francis, Elsevier's Science Direct, and Google Scholar.

\section{FINDINGS AND DISCUSSION}

Our study aimed at a literature review of prior studies on the impact of corporate sustainability reporting practice on corporate performance. Upon thorough review of the 35 works of literature, our study found 13 works of literature with positive relationship, 8 revealed negative association, 5 showed no significant relationship and finally, 9 showed mixed results. Consequently, our review of works of literature showed that sustainability indicators (economic, social, and governance factors) have unpredictable effects on the different performance measures. Notwithstanding the lack of consensus in literature, majority of the studies suggested a positive impact. This signifies sustainability practice will enhance corporate performance and aid competitive advantages for companies in the long run even though some costs will have to be borne in the short-term. The benefits that will accrue to firms will definitely outweigh the short-term cost only if firms can persevere. This study, therefore, organize reviewed literature based on their findings which range from positive results to negative, no significant relationship to mixed findings. This is pertinent to bring clarity of purpose and to ease understanding of the nature of relationship existing between the concept of sustainability reporting practice and corporate performance.

Table 1: Studies with positive relationship between sustainability reporting practice and corporate performance

\begin{tabular}{|c|c|c|c|c|c|c|}
\hline $\begin{array}{l}\mathrm{S} / \\
\mathrm{N}\end{array}$ & $\begin{array}{l}\text { Literature/ } \\
\text { Country }\end{array}$ & Proxy for SR & $\begin{array}{l}\text { Proxy for } \\
\text { CP }\end{array}$ & $\begin{array}{l}\text { Methodology and } \\
\text { Data sources }\end{array}$ & Theory & $\begin{array}{ll}\text { Findings, } & \text { Comments } \\
\text { Limitations } & \end{array}$ \\
\hline 1 & $\begin{array}{l}\text { Buallay } \\
\text { (2019) EU } \\
\text { (Develope } \\
\text { d country) }\end{array}$ & $\begin{array}{l}\text { Environment } \\
\text { al, social and } \\
\text { governance } \\
\text { (ESG) } \\
\text { disclosure }\end{array}$ & $\begin{array}{l}\text { Return on } \\
\text { Assets } \\
\text { (ROA), } \\
\text { Return on } \\
\text { Equity } \\
\text { (ROE) and } \\
\text { Tobin's Q } \\
\text { Control } \\
\text { variable: } \\
\text { GDP, } \\
\text { governanc } \\
\text { e (GOV), } \\
\text { total assets } \\
\text { and }\end{array}$ & $\begin{array}{l}\text { Sample consisted } \\
\text { of } 2,350 \\
\text { observations from } \\
235 \text { banks listed } \\
\text { on the European } \\
\text { Union (EU) stock } \\
\text { exchange over a } \\
\text { period of } 10 \text { years. } \\
\text { Data were sourced } \\
\text { from Bloomberg } \\
\text { database. }\end{array}$ & $\begin{array}{l}\text { Cost of } \\
\text { capital } \\
\text { theory; } \\
\text { Anticipatio } \\
\mathrm{n} \text { theory; } \\
\text { and } \\
\text { Instrument } \\
\text { al theory. }\end{array}$ & $\begin{array}{l}\text { The study examined relationship } \\
\text { between sustainability reporting and } \\
\text { performance for listed banks on the } \\
\text { EU stock exchange. Result revealed } \\
\text { ESG has a significant positive } \\
\text { impact on performance. However, } \\
\text { author recommend the EU banks to } \\
\text { focus more on sustainability } \\
\text { reporting for transparency of long- } \\
\text { term economic situation and non- } \\
\text { financial information; they also } \\
\text { recommended financial authority to } \\
\text { have clear and mandatory laws on } \\
\text { sustainability reporting because }\end{array}$ \\
\hline
\end{tabular}




\begin{tabular}{|c|c|c|c|c|c|c|}
\hline $\begin{array}{l}\mathrm{S} / \\
\mathrm{N}\end{array}$ & $\begin{array}{l}\text { Literature/ } \\
\text { Country }\end{array}$ & Proxy for SR & $\begin{array}{l}\text { Proxy for } \\
\mathrm{CP}\end{array}$ & $\begin{array}{l}\text { Methodology and } \\
\text { Data sources }\end{array}$ & Theory & $\begin{array}{l}\text { Findings, } \quad \text { Comments and } \\
\text { Limitations }\end{array}$ \\
\hline & & & $\begin{array}{l}\text { financial } \\
\text { leverage. }\end{array}$ & & & $\begin{array}{l}\text { existing laws are weak. Meanwhile, } \\
\text { the study only considered listed } \\
\text { banks in EU. }\end{array}$ \\
\hline 2 & $\begin{array}{l}\text { Emeka- } \\
\text { Nwokeji } \\
\& \text { Okeke } \\
(2019) \\
\text { Nigeria } \\
\text { (Developi } \\
\text { ng } \\
\text { country) }\end{array}$ & $\begin{array}{l}\text { Dummy } \\
\text { variables of } \\
\text { ' } 1 \text { ' and ' } 0 \text { ' } \\
\text { were } \\
\text { assigned to } \\
\text { quantitative } \\
\text { values of all } \\
\text { qualitative } \\
\text { specific } \\
\text { environment } \\
\text { al disclosure. }\end{array}$ & $\begin{array}{l}\text { Return on } \\
\text { Assets } \\
\text { (ROA) } \\
\text { Control } \\
\text { variables: } \\
\text { Firm size, } \\
\text { Age and } \\
\text { leverage. }\end{array}$ & $\begin{array}{l}\text { Based on ordinary } \\
\text { least square } \\
\text { regression, with } \\
\text { ex-post facto } \\
\text { research design } \\
\text { and content } \\
\text { analysis of annual } \\
\text { report for } 93 \text { non- } \\
\text { financial listed } \\
\text { firms on the } \\
\text { Nigerian Stock } \\
\text { Exchange } \\
\text { between } 2006 \text { to } \\
\text { 2015. Study data } \\
\text { were analyzed } \\
\text { with the aid of } \\
\text { STATA. }\end{array}$ & $\begin{array}{l}\text { Legitimacy } \\
\text { theory and } \\
\text { Agency } \\
\text { theory }\end{array}$ & $\begin{array}{l}\text { The study examined the effect of } \\
\text { environmental sustainability } \\
\text { disclosures on performance of firms } \\
\text { in Nigeria. Overall result revealed } \\
\text { aggregate environmental disclosures } \\
\text { had significant positive effects on } \\
\text { firm performance. However, when } \\
\text { the result were analyzed } \\
\text { individually, only environmental } \\
\text { compliance policy and disclosure of } \\
\text { environmental donation showed } \\
\text { significant positive effect while } \\
\text { energy consumption had significant } \\
\text { negative effect; environmental } \\
\text { sensitive products and } \\
\text { environmental anservative } \\
\text { disclosure had positive insignificant } \\
\text { effect on firm performance. Based } \\
\text { on this, the author advised that as a } \\
\text { matter of priority, firms should } \\
\text { adopt and disclose environmentally } \\
\text { friendly policies like making } \\
\text { donation towards environmental } \\
\text { protection, avoiding pollution and } \\
\text { hazardous wastes to the } \\
\text { environment. They believed doing } \\
\text { so would assist the firms to gain } \\
\text { social legitimacy that will enable } \\
\text { them to enjoy increase patronage } \\
\text { and revenue. } \\
\text { Study limitation was in the proxy } \\
\text { used for performance i.e. only ROA } \\
\text { which is an accounting based } \\
\text { measure, hence might not be } \\
\text { reflective of actual performance. }\end{array}$ \\
\hline 3 & $\begin{array}{l}\text { Jan, } \\
\text { Marimuth } \\
\text { u, bin } \\
\text { Mohd, and } \\
\text { Isa (2019) } \\
\text { Malaysia } \\
\text { (Developi } \\
\text { ng } \\
\text { country) }\end{array}$ & $\begin{array}{l}4 \\
\text { independent } \\
\text { variables } \\
\text { consisting of } \\
\text { general } \\
\text { standards } \\
\text { sustainabilit } \\
\text { y disclosers; } \\
\text { economic } \\
\text { sustainabilit } \\
\text { y; } \\
\text { environment } \\
\text { al } \\
\text { sustainabilit } \\
\text { y; and social } \\
\text { sustainabilit } \\
\text { y. }\end{array}$ & $\begin{array}{l}\text { Return on } \\
\text { average } \\
\text { assets } \\
\text { (ROAA); } \\
\text { Return on } \\
\text { average } \\
\text { equity } \\
\text { (ROAE); } \\
\text { Tobin's Q } \\
\text { and } \\
\text { Principal } \\
\text { Componen } \\
\text { t Analysis } \\
\text { (PCA). }\end{array}$ & $\begin{array}{l}\text { Using a weighted } \\
\text { content analysis, } \\
\text { all } 16 \text { Islamic } \\
\text { banks operating in } \\
\text { Malaysia were } \\
\text { sampled and data } \\
\text { was collected for } \\
\text { the post-crisis } \\
\text { period of 2009- } \\
2017 \text { from the } \\
\text { annual reports. }\end{array}$ & $\begin{array}{l}\text { Slack } \\
\text { resource } \\
\text { theory; } \\
\text { stakeholde } \\
\mathrm{r} \text { theory } \\
\text { and } \\
\text { Maqasid- } \\
\text { al-Shariah } \\
\text { theory. }\end{array}$ & $\begin{array}{l}\text { The particular study analyzed the } \\
\text { nexus between sustainability } \\
\text { practices and financial performance } \\
\text { for Islamic banks in Malaysia. } \\
\text { Findings revealed significant } \\
\text { positive association with the } \\
\text { financial performance. Study } \\
\text { recommendation is for management, } \\
\text { shareholders and market investors to } \\
\text { channel efforts to improve and } \\
\text { safeguard sustainability since it will } \\
\text { no doubt add financial values to the } \\
\text { stakeholders and the market profile } \\
\text { of the Islamic banks. } \\
\text { Notwithstanding, the study } \\
\text { limitation was its focus on only } \\
\text { Islamic banks in Malaysia. Hence, } \\
\text { findings cannot be generalized for } \\
\text { all the banks. }\end{array}$ \\
\hline 4 & $\begin{array}{l}\text { Borges } \\
\text { Junior } \\
(2019) \\
\text { Brazil } \\
\end{array}$ & $\begin{array}{l}\text { Dummy } \\
\text { variable for } \\
\text { publication } \\
\text { of }\end{array}$ & $\begin{array}{l}\text { Return on } \\
\text { assets } \\
\text { ROA, } \\
\text { company } \\
\end{array}$ & $\begin{array}{l}\text { Using descriptive } \\
\text { measure } \\
\text { correlation } \\
\text { analysis, data } \\
\end{array}$ & $\begin{array}{l}\text { Legitimacy } \\
\text { theory and } \\
\text { stakeholde } \\
\text { r theory. }\end{array}$ & $\begin{array}{l}\text { This study analyzed the association } \\
\text { between the publication of } \\
\text { sustainability report and } \\
\text { performance of Brazilian public }\end{array}$ \\
\hline
\end{tabular}




\begin{tabular}{|c|c|c|c|c|c|c|}
\hline S/ & $\begin{array}{l}\text { Literature/ } \\
\text { Country }\end{array}$ & Proxy for SR & $\begin{array}{l}\text { Proxy for } \\
\text { CP }\end{array}$ & $\begin{array}{l}\text { Methodology and } \\
\text { Data sources }\end{array}$ & Theory & $\begin{array}{lll}\begin{array}{l}\text { Findings, } \\
\text { Limitations }\end{array} & \text { Comments } & \text { and } \\
\end{array}$ \\
\hline & $\begin{array}{l}\text { (Developi } \\
\text { ng } \\
\text { country) }\end{array}$ & $\begin{array}{l}\text { sustainabilit } \\
\text { y report }\end{array}$ & $\begin{array}{l}\text { size and } \\
\text { capital } \\
\text { structure } \\
\text { (leverage) }\end{array}$ & $\begin{array}{l}\text { were collected on } \\
\text { the publication of } \\
\text { the sustainability } \\
\text { report for all non- } \\
\text { financial publicly } \\
\text { listed Brazilian } \\
\text { firms for the } \\
\text { period 2012-2016. }\end{array}$ & & $\begin{array}{l}\text { firms. Result showed positive and } \\
\text { statistically significant association } \\
\text { between the publication of the } \\
\text { sustainability report and } \\
\text { performance, size and leverage of } \\
\text { the company. This implies large, } \\
\text { profitable and highly leveraged } \\
\text { companies do have more resources } \\
\text { available to invest in voluntary } \\
\text { reporting. This study limitation was } \\
\text { in the fact that it focused only on } \\
\text { company responses recorded on } \\
\text { publication of sustainability report } \\
\text { and examined only a single country. }\end{array}$ \\
\hline 5 & $\begin{array}{l}\text { Zhao et al. } \\
\text { (2018) } \\
\text { China } \\
\text { (Developi } \\
\text { ng } \\
\text { country) }\end{array}$ & $\begin{array}{l}\text { ESG } \\
\text { indicators } \\
\text { developed } \\
\text { based on } \\
\text { PSR concept }\end{array}$ & $\begin{array}{l}\text { Return on } \\
\text { capital } \\
\text { employed } \\
\text { (ROCE), } \\
\text { debt-to- } \\
\text { equity ratio } \\
\text { (D/E) and } \\
\text { logarithm } \\
\text { of total } \\
\text { assets (Log } \\
\text { TA) for } \\
\text { Size. }\end{array}$ & $\begin{array}{l}\text { Sample consisted } \\
\text { of } 20 \text { large listed } \\
\text { power generation } \\
\text { companies from } 5 \\
\text { major China } \\
\text { Resources Power } \\
\text { Holdings } \\
\text { Company. Data } \\
\text { were sourced from } \\
\text { the CSMAR for a } \\
\text { period of } 10 y e a r s .\end{array}$ & $\begin{array}{l}\text { Pressure } \\
\text { state } \\
\text { response } \\
\text { (PSR) } \\
\text { concept }\end{array}$ & $\begin{array}{l}\text { The study investigated impact of } \\
\text { ESG on performance and found } \\
\text { good ESG performance will } \\
\text { improve financial performance. } \\
\text { Author suggested strengthening the } \\
\text { construction of CSR standards will } \\
\text { have a long-term and outstanding } \\
\text { contribution for company's financial } \\
\text { performance. } \\
\text { Study limitation was in the area of } \\
\text { methodology used. Proxy used for } \\
\text { sustainability reporting was too } \\
\text { ambiguous and confusing, and only } \\
\text { focus listed power generation } \\
\text { companies in China. }\end{array}$ \\
\hline 6 & $\begin{array}{l}\text { Garcia, } \\
\text { Mendes- } \\
\text { Da-Silva, } \\
\text { and } \\
\text { Orsato } \\
\text { (2017) } \\
\text { Brazil, } \\
\text { Russia, } \\
\text { India, } \\
\text { China and } \\
\text { South } \\
\text { Africa } \\
\text { (BRICS) } \\
\text { (Developi } \\
\text { ng } \\
\text { countries) }\end{array}$ & $\begin{array}{l}\text { Economic, } \\
\text { Social and } \\
\text { Governance } \\
\text { scores and } \\
\text { Overall ESG } \\
\text { scores from } \\
\text { Thomson } \\
\text { Reuters. }\end{array}$ & $\begin{array}{l}\text { Systematic } \\
\text { risk index, } \\
\text { financial } \\
\text { leverage } \\
\text { index, free } \\
\text { cash flow, } \\
\text { market } \\
\text { capitalizati } \\
\text { on, Return } \\
\text { on assets } \\
\text { (ROA), } \\
\text { firm size } \\
\text { and sector } \\
\text { dummy. }\end{array}$ & $\begin{array}{l}\text { Based on a linear } \\
\text { regression panel } \\
\text { data analysis, data } \\
\text { were sourced for } \\
365 \text { BRICS non- } \\
\text { financial } \\
\text { companies over } \\
\text { the period } 2010- \\
2012 \text { from } \\
\text { Thomson Reuters } \\
\text { and DataStream }\end{array}$ & $\begin{array}{l}\text { Stakeholde } \\
\mathrm{r} \text { theory } \\
\text { and } \\
\text { legitimacy } \\
\text { theory. }\end{array}$ & $\begin{array}{l}\text { Study examined firm risk and ESG } \\
\text { performance. Result indicated an } \\
\text { inverted U-shaped curve, signifying } \\
\text { the existence of a maximum value } \\
\text { for ESG performance through the } \\
\text { firm's systematic risk level. Result } \\
\text { equally indicated firms operating in } \\
\text { highly sensitive industries do } \\
\text { present superior environmental } \\
\text { performance than others because } \\
\text { their activities have high tendency to } \\
\text { impose damage on the society. } \\
\text { Limitation of this study was it only } \\
\text { considered companies from the } \\
\text { BRICS countries; and the } \\
\text { econometric technique used could } \\
\text { be subject to bias. }\end{array}$ \\
\hline 7 & $\begin{array}{l}\text { Loh, } \\
\text { Thomas, } \\
\text { and Wang } \\
\text { (2017) } \\
\text { Singapore } \\
\text { (Developi } \\
\text { ng } \\
\text { country) }\end{array}$ & $\begin{array}{l}4 \text { indicators } \\
\text { of } \\
\text { Governance, } \\
\text { Economic, } \\
\text { Environment } \\
\text { al and Social. }\end{array}$ & $\begin{array}{l}4 \quad \text { months } \\
\text { market } \\
\text { value; } \\
\text { control } \\
\text { variables: } \\
\text { governmen } \\
\text { t-linked } \\
\text { companies } \\
\text { (GLC), } \\
\text { family } \\
\text { business } \\
\text { (FB) and } \\
\text { high } \\
\text { impact } \\
\text { sector (HI) }\end{array}$ & $\begin{array}{l}\text { Using Ohlson } \\
\text { model based on } \\
\text { weighted least } \\
\text { square regression; } \\
\text { sample consist of } \\
502 \text { listed firms on } \\
\text { Singapore stock } \\
\text { exchange. Data } \\
\text { were sourced from } \\
\text { Bloomberg, Osiris } \\
\text { and company } \\
\text { disclosure up to } \\
2015 \text {. }\end{array}$ & $\begin{array}{l}\text { Agency } \\
\text { theory, } \\
\text { signaling } \\
\text { theory and } \\
\text { legitimacy } \\
\text { theory. }\end{array}$ & $\begin{array}{l}\text { Study examined linkage between } \\
\text { sustainability reporting and firm } \\
\text { value. Results showed that } \\
\text { sustainability disclosure is } \\
\text { positively related to the market } \\
\text { value of firms, even though they } \\
\text { found firm status such as } \\
\text { government ownership, family } \\
\text { business and operating in high } \\
\text { impact sectors having no relation. } \\
\text { Limitation of this study was that it } \\
\text { considered one country only and } \\
\text { variables used might have } \\
\text { influenced their result. }\end{array}$ \\
\hline
\end{tabular}




\begin{tabular}{|c|c|c|c|c|c|c|}
\hline $\begin{array}{l}\mathrm{S} / \\
\mathrm{N}\end{array}$ & $\begin{array}{l}\text { Literature/ } \\
\text { Country }\end{array}$ & Proxy for SR & $\begin{array}{l}\text { Proxy for } \\
\text { CP }\end{array}$ & $\begin{array}{l}\text { Methodology and } \\
\text { Data sources }\end{array}$ & Theory & $\begin{array}{l}\text { Findings, } \\
\text { Limitations }\end{array}$ \\
\hline 8 & $\begin{array}{l}\text { Tarmuji, } \\
\text { Maelah, } \\
\text { and } \\
\text { Tarmuji } \\
\text { (2016) } \\
\text { Malaysia } \\
\& \\
\text { Singapore } \\
\text { (Developi } \\
\text { ng } \\
\text { countries) }\end{array}$ & $\begin{array}{l}3 \text { indicators } \\
\text { of } \\
\text { Environment } \\
\text { al, } \\
\text { Governance } \\
\text { and Social. }\end{array}$ & $\begin{array}{l}\text { Economic } \\
\text { performan } \\
\text { ce }\end{array}$ & $\begin{array}{l}\text { Using Regression } \\
\text { analysis on a } \\
\text { sample of } 80 \\
\text { companies, } 35 \\
\text { from Malaysia and } \\
45 \text { from Singapore } \\
\text { for the period } \\
\text { between 2010- } \\
2014 \text {. }\end{array}$ & $\begin{array}{l}\text { Agency } \\
\text { theory and } \\
\text { stakeholde } \\
r \text { theory. }\end{array}$ & $\begin{array}{l}\text { The study investigated the impact of } \\
\text { ESG practices on economic } \\
\text { performance. Result showed } \\
\text { positive significant relationship for } \\
\text { all the variables on economic } \\
\text { performance. Study lapses relied on } \\
\text { the sample size which was relatively } \\
\text { small. Hence, results should not be } \\
\text { generalized. }\end{array}$ \\
\hline 9 & $\begin{array}{l}\text { Cornett, } \\
\text { Erhemjam } \\
\text { ts, and } \\
\text { Tehranian } \\
\text { (2016) US } \\
\text { (Develope } \\
\text { d) }\end{array}$ & $\begin{array}{l}\text { ESG ratings } \\
\text { based on } 35 \\
\text { indicators }\end{array}$ & $\begin{array}{l}\text { ROA, } \\
\text { ROE, } \\
\text { Tobin's Q, } \\
\text { operating } \\
\text { profit, } \\
\text { Size, } \\
\text { capital } \\
\text { levels, high } \\
\text { fees, board } \\
\text { compositio } \\
\text { n, external } \\
\text { political } \\
\text { environme } \\
\text { nt, }\end{array}$ & $\begin{array}{l}\text { Based on OLS } \\
\text { regression, study } \\
\text { examined } 235 \text { US } \\
\text { banks between } \\
2003-2013 \text { with } \\
\text { data collected } \\
\text { from MSCI ESG } \\
\text { STATS database. }\end{array}$ & $\begin{array}{l}\text { Social } \\
\text { network } \\
\text { theory. }\end{array}$ & $\begin{array}{l}\text { The article analyzed relationship } \\
\text { between banks' social performance } \\
\text { and financial performance and } \\
\text { findings reveal significant positive } \\
\text { relationship between ROE and CSR } \\
\text { scores. Findings showed that } \\
\text { collapse due to the recent financial } \\
\text { crisis was what influenced banks } \\
\text { and their stakeholders to intensify } \\
\text { efforts towards sustainable } \\
\text { practices. Meanwhile, the study still } \\
\text { had limitation since it focused only } \\
\text { on the US which is a developed } \\
\text { market. }\end{array}$ \\
\hline 10 & $\begin{array}{l}\text { Burhan } \\
\text { and } \\
\text { Rahmanti } \\
\text { (2012) } \\
\text { Indonesia } \\
\text { (Developi } \\
\text { ng } \\
\text { country) }\end{array}$ & $\begin{array}{l}\text { Economic, } \\
\text { Social and } \\
\text { Governance } \\
\text { performance } \\
\text { index based } \\
\text { on GRI } \\
\text { framework }\end{array}$ & $\begin{array}{l}\text { Return on } \\
\text { assets } \\
\text { (ROA) }\end{array}$ & $\begin{array}{lr}\begin{array}{lr}\text { Using a } \\
\text { regression }\end{array} & \begin{array}{r}\text { linear } \\
\text { for }\end{array} \\
\text { listed } & \text { non- } \\
\text { financial } & \\
\text { companies } & \text { in } \\
\text { Indonesia, } & \text { for } \\
2006-2009 . & \end{array}$ & $\begin{array}{l}\text { Legitimacy } \\
\text { theory and } \\
\text { stakeholde } \\
r \text { theory. }\end{array}$ & $\begin{array}{l}\text { Similar to prior study, this author } \\
\text { found only social performance } \\
\text { disclosure positively influenced } \\
\text { company performance. Author } \\
\text { therefore opined companies will } \\
\text { start acting responsibly since } \\
\text { without the credibility and trust put } \\
\text { by stakeholders, it would be difficult } \\
\text { for businesses to thrive well. } \\
\text { However, research focus covered } \\
\text { only a small sample of } 32 \text { companies } \\
\text { over a short period of time. }\end{array}$ \\
\hline 11 & $\begin{array}{l}\text { Ameer } \\
\text { and } \\
\text { Othman } \\
(2012) \\
\text { Multi } \\
\text { country }\end{array}$ & $\begin{array}{l}\text { Sustainabilit } \\
\text { y Index } \\
\text { scores } \\
\text { covering } \\
\text { Environment } \\
\text { Diversity, } \\
\text { Community } \\
\text { and Ethical } \\
\text { standards. } \\
\text { The item } \\
\text { were scored } \\
\text { 0-4 based on } \\
\text { disclosure in } \\
\text { sustainabilit } \\
\text { y report. }\end{array}$ & $\begin{array}{l}\text { Sales } \\
\text { revenue } \\
\text { growth } \\
\text { (SRG), } \\
\text { Return on } \\
\text { assets } \\
\text { (ROA), } \\
\text { Profit } \\
\text { before tax } \\
\text { (PBT), and } \\
\text { cash flows } \\
\text { from } \\
\text { operating } \\
\text { activities } \\
\text { (CFO). }\end{array}$ & $\begin{array}{l}\text { Sample consisted } \\
\text { of Top } 100 \\
\text { sustainable global } \\
\text { companies for the } \\
\text { period 2006-2010. } \\
\text { ESG data was } \\
\text { drawn using } \\
\text { content analysis of } \\
\text { sustainability } \\
\text { reports while the } \\
\text { financial data } \\
\text { were sourced from } \\
\text { Thomson } \\
\text { financials World } \\
\text { scope. }\end{array}$ & Nil & $\begin{array}{l}\text { This study examined whether } \\
\text { companies with superior } \\
\text { sustainability practices have } \\
\text { superior financial performance and } \\
\text { growth than companies that do not. } \\
\text { Findings revealed firms with higher } \\
\text { sustainability disclosure scores had } \\
\text { significantly higher mean sales } \\
\text { revenue growth, ROA, PBT and } \\
\text { CFO over the period. examined. } \\
\text { Study limitation was on the sample } \\
\text { which were drawn from only the top } \\
100 \text { global sustainable companies } \\
\text { from US. }\end{array}$ \\
\hline 12 & $\begin{array}{l}\text { Reddy and } \\
\text { Gordon } \\
\text { (2010). } \\
\text { Australia } \\
\& \quad \text { New } \\
\text { Zealand } \\
\text { (Develope } \\
\text { d } \\
\text { country). }\end{array}$ & $\begin{array}{l}\text { Dummy } \\
\text { variables D1, } \\
\text { D2 \& D3 } \\
\text { equal 1 if the } \\
\text { sustainabilit } \\
\text { y report is of } \\
\text { the } \\
\text { correspondin }\end{array}$ & $\begin{array}{l}\text { Abnormal } \\
\text { returns }\end{array}$ & $\begin{array}{l}\text { Using event study, } \\
\text { sample included } \\
68 \quad \text { listed } \\
\text { companies, from } \\
\text { New Zealand and } \\
\text { Australian Stock } \\
\text { Exchange over } 31 \\
\text { days of market } \\
\text { announcement. }\end{array}$ & Nil & $\begin{array}{l}\text { The study found a statistically } \\
\text { significant relationship with market } \\
\text { returns for Australian companies } \\
\text { and a systematic positive } \\
\text { relationship for New Zealand. Upon } \\
\text { identifying several contextual } \\
\text { factors, such as industry and type of } \\
\text { sustainability report, having } \\
\text { potential to impact the relationship }\end{array}$ \\
\hline
\end{tabular}




\begin{tabular}{|c|c|c|c|c|c|c|}
\hline $\begin{array}{l}\mathrm{S} / \\
\mathrm{N}\end{array}$ & $\begin{array}{l}\text { Literature/ } \\
\text { Country }\end{array}$ & Proxy for SR & $\begin{array}{l}\text { Proxy for } \\
\mathrm{CP}\end{array}$ & $\begin{array}{l}\text { Methodology and } \\
\text { Data sources }\end{array}$ & Theory & $\begin{array}{lll}\text { Findings, } & \text { Comments } & \text { and } \\
\text { Limitations } & & \\
\end{array}$ \\
\hline & & $\begin{array}{l}\mathrm{g} \text { type, } \\
\text { otherwise } 0 .\end{array}$ & & & & $\begin{array}{l}\text { between sustainability reporting and } \\
\text { financial performance, study } \\
\text { limitation stood in the fact that it } \\
\text { considered only developed } \\
\text { economies. }\end{array}$ \\
\hline 13 & $\begin{array}{l}\text { Lin, Yang, } \\
\text { and Liou } \\
\text { (2009). } \\
\text { Taiwan } \\
\text { (Developi } \\
\text { ng } \\
\text { country) }\end{array}$ & $\begin{array}{l}\text { Donation } \\
\text { ratio as a } \\
\text { CSR proxy } \\
\text { variable. }\end{array}$ & $\begin{array}{l}\text { Return on } \\
\text { Asset } \\
(\mathrm{ROA}) \text { and } \\
\text { rate of } \\
\text { stock } \\
\text { return }\end{array}$ & $\begin{array}{l}\text { Sample consisted } \\
\text { of top } 1000 \\
\text { Taiwan-based } \\
\text { companies } \\
\text { Company } \\
\text { financial data was } \\
\text { retrieved from the } \\
\text { Taiwan Economic } \\
\text { Journal Databank } \\
\text { from 2002 to } \\
2004 \text {. }\end{array}$ & $\begin{array}{l}\text { Strategic } \\
\text { business } \\
\text { and } \\
\text { supply- } \\
\text { chain } \\
\text { perspective } \\
\text {. }\end{array}$ & $\begin{array}{l}\text { This study pointed to the need to } \\
\text { move social responsibility research } \\
\text { from bivariate relationships to a } \\
\text { more context-specific approach. The } \\
\text { study considered corporate } \\
\text { investment in R\&D, and result } \\
\text { showed positive relationship } \\
\text { between CSR and financial } \\
\text { performance in the long-term only. } \\
\text { Study limitation relied on the fact } \\
\text { that author used survey } \\
\text { methodology making the measure of } \\
\text { CSR not to be objective; and the } \\
\text { sample only considered large } \\
\text { manufacturing firms. The study also } \\
\text { failed to control for industry effect } \\
\text { that could have greatly influence the } \\
\text { relationship between variables. }\end{array}$ \\
\hline
\end{tabular}

Table 2: Studies with negative relationship between sustainability reporting practice and corporate performance

\begin{tabular}{|c|c|c|c|c|c|c|}
\hline $\begin{array}{l}\mathrm{S} / \\
\mathrm{N} \\
\mathrm{O}\end{array}$ & Literature & $\begin{array}{l}\text { Proxy for } \\
\text { SR }\end{array}$ & $\begin{array}{l}\text { Proxy for } \\
\text { CP }\end{array}$ & $\begin{array}{l}\text { Methodology } \\
\text { and Data sources }\end{array}$ & Theory & $\begin{array}{l}\text { Findings, reason for contrary opinion and } \\
\text { Limitations }\end{array}$ \\
\hline 1 & $\begin{array}{l}\text { Dinçer and } \\
\text { Altınay } \\
(2020) \\
\text { Turkey } \\
\text { (Developing } \\
\text { ) }\end{array}$ & $\begin{array}{l}4 \\
\text { Indicators } \\
\text { of } \\
\text { environme } \\
\text { nt, human } \\
\text { resources, } \\
\text { product } \\
\text { liability, } \\
\text { and } \\
\text { community } \\
\text { involveme } \\
\text { nt. }\end{array}$ & $\begin{array}{l}\text { Return } \\
\text { on assets } \\
\text { (ROA), } \\
\text { Return } \\
\text { on equity } \\
\text { (ROE), } \\
\text { Net } \\
\text { interest } \\
\text { margin } \\
\text { (NIM). }\end{array}$ & $\begin{array}{l}\text { Using a scoring } \\
\text { model, } 7 \text { banks } \\
\text { were selected } \\
\text { from banks } \\
\text { listed on Bursa } \\
\text { Istanbul, Turkey } \\
\text { (BIST) } \\
\text { Sustainability } \\
\text { Index between } \\
\text { 2010-2017. Data } \\
\text { were sourced } \\
\text { from banks } \\
\text { sustainability } \\
\text { reports. }\end{array}$ & $\begin{array}{l}\text { Institutio } \\
\text { nal } \\
\text { theory. }\end{array}$ & $\begin{array}{l}\text { Study analyzed the effect of banks' } \\
\text { sustainability reports' declarations on } \\
\text { financial performance, but result showed } \\
\text { negative impact. Plausible explanation for } \\
\text { contrary findings are that bank's standards } \\
\text { are continually updated and requires them } \\
\text { to be disciplined, hence, banks result to } \\
\text { using reporting on environmental issue as } \\
\text { an advertisement/promotion tool for } \\
\text { prestige; adopting sustainability practice } \\
\text { require making changes in business } \\
\text { techniques. For this reason, shareholders } \\
\text { see it as a cost object the imposes extra } \\
\text { cost for the banks. Study limitation } \\
\text { stemmed from the small sample and small } \\
\text { period covered. }\end{array}$ \\
\hline 2 & $\begin{array}{l}\text { Rajesh and } \\
\text { Rajendran, } \\
(2020) \\
\text { Country not } \\
\text { stated }\end{array}$ & $\begin{array}{l}\text { Sustainabil } \\
\text { ity } \\
\text { performan } \\
\text { ce was } \\
\text { based on } \\
\text { publicly } \\
\text { reported } \\
\text { informatio } \\
\text { n }\end{array}$ & $\begin{array}{l}\text { ESG } \\
\text { scores } \\
\text { from } \\
\text { Thomson } \\
\text { Reuters } \\
\text { based on } \\
10 \\
\text { indicator } \\
\text { s. }\end{array}$ & $\begin{array}{l}\text { Partial Least } \\
\text { Square (PLS) } \\
\text { analysis based } \\
\text { on Smart PLS } \\
\text { 3.0, was used to } \\
\text { evaluate the } \\
\text { measurement of } \\
\text { the structural } \\
\text { models. Final } \\
\text { sample } \\
\text { consisted } \\
\text { periodic data of } \\
\text { 1820 firms over } \\
\text { 5years from } \\
2009 \text { to } 2018 \text {. }\end{array}$ & $\begin{array}{l}\text { Ecologic } \\
\text { al } \\
\text { moderniz } \\
\text { ation } \\
\text { theory; } \\
\text { Continge } \\
\text { ncy } \\
\text { theory; } \\
\text { Institutio } \\
\text { nal } \\
\text { theory. }\end{array}$ & $\begin{array}{l}\text { This study observed a significant negative } \\
\text { moderating effect of ESG performances } \\
\text { on sustainability performance. Author } \\
\text { therefore adviced managers to study in- } \\
\text { depth the moderating effects of each of } \\
\text { environmental, social and governance } \\
\text { (ESG) performances to observe how far } \\
\text { the individual effects can improve the } \\
\text { overall sustainability performances for } \\
\text { firms. Study however, pointed to the fact } \\
\text { that ESG performances may be guided } \\
\text { through different pressures from } \\
\text { stakeholders, customers, competitors, and } \\
\text { governments. Reason for contrary result } \\
\text { stemmed from the argument that when } \\
\text { pressure from stakeholders keeps }\end{array}$ \\
\hline
\end{tabular}




\begin{tabular}{|c|c|c|c|c|c|c|}
\hline $\begin{array}{l}\mathrm{S} / \\
\mathrm{N} \\
\mathrm{O}\end{array}$ & Literature & $\begin{array}{l}\text { Proxy for } \\
\text { SR }\end{array}$ & $\begin{array}{l}\text { Proxy for } \\
\text { CP }\end{array}$ & $\begin{array}{l}\text { Methodology } \\
\text { and Data sources }\end{array}$ & Theory & $\begin{array}{l}\text { Findings, reason for contrary opinion and } \\
\text { Limitations }\end{array}$ \\
\hline & & & & & & $\begin{array}{l}\text { mounting for increased visibility, firms' } \\
\text { focus, and their supply chain would } \\
\text { improve towards achieving different } \\
\text { dimensions of sustainability } \\
\text { performances. Study limitation was, no } \\
\text { doubt, in the direction taken to assign } \\
\text { priorities to environmental, social, and } \\
\text { governance related themes in the } \\
\text { implementation of strategies and for the } \\
\text { fact that the study failed to consider effect } \\
\text { of control variables. }\end{array}$ \\
\hline 3 & $\begin{array}{l}\text { Alcaide } \\
\text { González, } \\
\text { De La Poza } \\
\text { Plaza, and } \\
\text { Guadalajara } \\
\text { Olmeda } \\
\text { (2020) } \\
\text { Global } 100\end{array}$ & $\begin{array}{l}\text { ESG } \\
\text { Scores in } \\
\text { Reptrak; } \\
\text { Global } \\
\text { 100, Green } \\
\text { ranking; } \\
\text { Finance } \\
\text { yahoo } \\
\text { sustainabili } \\
\text { ty; } \\
\text { Interbrand; } \\
\text { brand } \\
\text { finance; } \\
\text { Millward } \\
\text { brown. }\end{array}$ & $\begin{array}{l}\text { Size, } \\
\text { increase } \\
\text { in total } \\
\text { assets, } \\
\text { increase } \\
\text { in } \\
\text { revenues, } \\
\text { leverage, } \\
\text { ROE, } \\
\text { ROA and } \\
\text { number } \\
\text { of } \\
\text { employee } \\
\text { s in each } \\
\text { tax year. }\end{array}$ & $\begin{array}{l}\text { Using a } \\
\text { multivariate } \\
\text { linear regression } \\
\text { by ordinary least } \\
\text { squares for a } \\
\text { sample of } 13 \\
\text { companies in the } \\
\text { IT sector from } \\
\text { top } 100 \text { global } \\
\text { ranking for the } \\
\text { period 2000- } \\
2018\end{array}$ & Nil & $\begin{array}{l}\text { While the study examined relationship } \\
\text { between ESG scores and firm } \\
\text { performance, result showed that although } \\
\text { large companies are more transparent in } \\
\text { terms of sustainability, it does not relate } \\
\text { to their financial behavior. In essence, } \\
\text { findings indicated that if companies in the } \\
\text { technology sector achieve more } \\
\text { transparency and certain standards to } \\
\text { prepare sustainability reports, it will serve } \\
\text { as an incentive to increase the value of } \\
\text { their brands, particularly the value of their } \\
\text { intangible assets will become increasingly } \\
\text { more relevant compared to other sectors. } \\
\text { This study had limitation with regards to } \\
\text { the sample size and the period covered by } \\
\text { the analysis. }\end{array}$ \\
\hline 4 & $\begin{array}{l}\text { Fatemi et al. } \\
\text { (2018) US } \\
\text { (Developed) }\end{array}$ & $\begin{array}{l}\text { ESG } \\
\text { performan } \\
\text { ce proxied } \\
\text { by ESG } \\
\text { strength } \\
\text { and ESG } \\
\text { concern. } \\
\text { Moderator: } \\
\text { ESG } \\
\text { disclosure. }\end{array}$ & $\begin{array}{l}\text { Tobin's } \\
\text { Q, } \\
\text { Control } \\
\text { variables: } \\
\text { Return } \\
\text { on Asset, } \\
\text { Growth } \\
\text { of Return } \\
\text { on assets, } \\
\text { firm size, } \\
\text { asset } \\
\text { intensity, } \\
\text { leverage. } \\
\text { advertisi } \\
\text { ng } \\
\text { intensity, } \\
\text { R\&D } \\
\text { expendit } \\
\text { ures, and } \\
\text { asset age. }\end{array}$ & $\begin{array}{l}\text { Using } \\
\text { Regression } \\
\text { analysis based } \\
\text { on data sourced } \\
\text { from KLD and } \\
\text { Bloomberg for } \\
403 \text { U.S. listed } \\
\text { companies for } \\
\text { the period } 2006 \\
-2011 .\end{array}$ & $\begin{array}{l}\text { Neoclass } \\
\text { ical } \\
\text { theory, } \\
\text { Stakehol } \\
\text { der } \\
\text { theory, } \\
\text { voluntary } \\
\text { disclosur } \\
\text { e theory. }\end{array}$ & $\begin{array}{l}\text { Similar to previous study above, findings } \\
\text { from this study indicated ESG strength } \\
\text { increases firm value while ESG concern } \\
\text { decreases it, and in overall, high ESG } \\
\text { disclosure weakens the positive valuation } \\
\text { effect of ESG strengths. Reason expunge } \\
\text { for the findings may be due to the markets } \\
\text { interpreting stepped-up disclosure as the } \\
\text { firm's attempt to justify their over } \\
\text { investment in ESG activities. While the } \\
\text { disclosure which was found to be } \\
\text { weakening the negative valuation effects } \\
\text { of ESG concerns, could be because } \\
\text { disclosures help firms legitimate their } \\
\text { behavior by explaining to investors the } \\
\text { appropriateness of their operations or } \\
\text { because firms convince investors that they } \\
\text { have made credible commitments to } \\
\text { change their operations and thus } \\
\text { overcome ESG weaknesses. Study } \\
\text { limitation may be due to its focus on only } \\
\text { US listed firms. }\end{array}$ \\
\hline
\end{tabular}




\begin{tabular}{|c|c|c|c|c|c|c|}
\hline S/ & terature & $\begin{array}{l}\text { Proxy for } \\
\text { SR }\end{array}$ & $\begin{array}{l}\text { Proxy for } \\
\text { CP }\end{array}$ & $\begin{array}{l}\text { Methodology } \\
\text { and Data sources }\end{array}$ & Theory & $\begin{array}{l}\text { Findings, reason for contrary opinion and } \\
\text { Limitations }\end{array}$ \\
\hline & $\begin{array}{l}\text { Abba, Said, } \\
\text { Abdullah, } \\
\text { and Mahat } \\
\text { (2018) } \\
\text { Nigeria } \\
\text { (Developing } \\
\text { ) }\end{array}$ & $\begin{array}{l}\text { Environme } \\
\text { ntal } \\
\text { disclosure } \\
\text { (ED), } \\
\text { environme } \\
\text { ntal } \\
\text { disclosure } \\
\text { level } \\
\text { (EDL) and } \\
\text { environme } \\
\text { ntal } \\
\text { disclosure } \\
\text { quality } \\
\text { (EDQ). }\end{array}$ & $\begin{array}{l}\text { Environ } \\
\text { mental } \\
\text { operation } \\
\text { al } \\
\text { performa } \\
\text { nce } \\
\text { Control } \\
\text { variable: } \\
\text { size, } \\
\text { profitabil } \\
\text { ity, } \\
\text { leverage, } \\
\text { regulator } \\
\text { y } \\
\text { pressure, } \\
\text { competiti } \\
\text { ve } \\
\text { strategy, } \\
\text { and audit } \\
\text { quality. }\end{array}$ & $\begin{array}{l}\text { Using content } \\
\text { analysis, the } \\
\text { study examined } \\
\text { manufacturing } \\
\text { industry listed } \\
\text { on Nigerian } \\
\text { Stock Exchange } \\
\text { Market (NSE). } \\
\text { Final sample } \\
\text { consisted of } 53 \\
\text { companies }\end{array}$ & $\begin{array}{l}\text { Voluntar } \\
\text { y } \\
\text { disclosur } \\
\text { e theory } \\
\text { and } \\
\text { legitimac } \\
\text { y theory. }\end{array}$ & $\begin{array}{l}\text { This particular study found no statistical } \\
\text { evidence to support the relationship } \\
\text { between performance and disclosure } \\
\text { quality. Result showed that } \\
\text { manufacturing companies in Nigeria use } \\
\text { the disclosure to legitimatize their } \\
\text { existence in the society. Moreover, they } \\
\text { used the tactics to maintain, and sway } \\
\text { public opinion about their environmental } \\
\text { performance. This study contributed to } \\
\text { the understanding of the green-wash issue } \\
\text { about environmental disclosure. The } \\
\text { study unveiled non-appreciation of } \\
\text { disclosure quality by firms to achieve } \\
\text { selection preference. Study limitation } \\
\text { hinged on the fact that only } \\
\text { manufacturing firms were considered and } \\
\text { focus on small sample size. }\end{array}$ \\
\hline 6 & $\begin{array}{l}\text { Joshi, } \\
\text { Pandey, and } \\
\text { Ross (2017) } \\
\text { US } \\
\text { (Developed) }\end{array}$ & $\begin{array}{l}\text { Dow Jones } \\
\text { Sustainabil } \\
\text { ity Index } \\
\text { (DJSI)/SA } \\
\text { M }\end{array}$ & $\begin{array}{l}\text { Stock } \\
\text { Returns }\end{array}$ &  & $\begin{array}{l}\text { Neo } \\
\text { classical } \\
\text { theory, } \\
\text { Instrume } \\
\text { ntal } \\
\text { stakehold } \\
\text { er theory, } \\
\text { Resource } \\
\text { based } \\
\text { view, } \\
\text { Institutio } \\
\text { nal } \\
\text { theory } \\
\text { and } \\
\text { tourname } \\
\text { nt theory. }\end{array}$ & $\begin{array}{l}\text { This study slightly differed from the } \\
\text { previous in that they used event study } \\
\text { methodology, but overall result showed } \\
\text { negative reaction to inclusion on DJSI. } \\
\text { The results suggested that markets on } \\
\text { average reacted negatively to DJSI } \\
\text { inclusion and non-positively to exclusion. } \\
\text { However, controversy could be because } \\
\text { investors perceive firm's addition to the } \\
\text { DJSI and winning such sustainability } \\
\text { leadership tournaments as shareholder } \\
\text { value destroying i.e. considerations such } \\
\text { as the potential additional constraints on } \\
\text { production technology, over-compliance } \\
\text { resulting in competitive disadvantage, } \\
\text { and diversion of managerial attention and } \\
\text { resources from productivity improvement } \\
\text { to been overshadowed by considerations } \\
\text { of potential avenues through which } \\
\text { sustainability efforts would have added to } \\
\text { the firm value; or probably because } \\
\text { investor planning horizons was relatively } \\
\text { too short. This study also failed to } \\
\text { consider other economies as it focused } \\
\text { only on US firms listed on the Dow jones } \\
\text { sustainability index. }\end{array}$ \\
\hline 7 & $\begin{array}{l}\text { Detre and } \\
\text { Gunderson } \\
\text { (2011) US } \\
\text { (Developed) }\end{array}$ & $\begin{array}{l}\text { Announce } \\
\text { ment for } \\
\text { firm's } \\
\text { inclusion } \\
\text { in DJSI }\end{array}$ & $\begin{array}{l}\text { Share } \\
\text { values } \\
\text { and } \\
\text { cumulati } \\
\text { ve } \\
\text { abnormal } \\
\text { returns } \\
\text { (CAR). }\end{array}$ & $\begin{array}{l}\text { Using an event } \\
\text { study method, } \\
\text { sample } \\
\text { consisted of } 36 \\
\text { publicly traded } \\
\text { US agribusiness } \\
\text { whor are } \\
\text { members of } \\
\text { DJSI and traded } \\
\text { on NYSE, } \\
\text { NASDAQ or } \\
\text { AMEX. }\end{array}$ & Nil & $\begin{array}{l}\text { This study applied a similar approach as } \\
\text { Joshi, Pandey, and Ross (2017) since they } \\
\text { used an event study methods. Meanwhile, } \\
\text { their result revealed agribusiness react } \\
\text { negatively and significantly only in the } \\
\text { short term when announcements are } \\
\text { made. This could be due to increased } \\
\text { costs that is often associated with } \\
\text { sustainable initiatives when initially } \\
\text { implemented. The study however failed to } \\
\text { distinguish between the agribusiness } \\
\text { firms to show their level of commitment } \\
\text { to the dimensions of sustainability. }\end{array}$ \\
\hline
\end{tabular}




\begin{tabular}{|c|c|c|c|c|c|c|}
\hline $\begin{array}{l}\mathrm{S} / \\
\mathrm{N} \\
\mathrm{O}\end{array}$ & Literature & $\begin{array}{l}\text { Proxy for } \\
\text { SR }\end{array}$ & $\begin{array}{l}\text { Proxy for } \\
\text { CP }\end{array}$ & $\begin{array}{l}\text { Methodology } \\
\text { and Data sources }\end{array}$ & Theory & $\begin{array}{l}\text { Findings, reason for contrary opinion and } \\
\text { Limitations }\end{array}$ \\
\hline 8 & $\begin{array}{l}\text { Jones, Frost, } \\
\text { Loftus, and } \\
\text { Van Der } \\
\text { Laan (2007) } \\
\text { Australia } \\
\text { (Developed) }\end{array}$ & $\begin{array}{l}\text { GRI } \\
\text { guidelines } \\
\text { for ESG } \\
\text { performan } \\
\text { ce }\end{array}$ & $\begin{array}{l}\text { Cash } \\
\text { position, } \\
\text { cashflow, } \\
\text { working } \\
\text { capital, } \\
\text { profitabil } \\
\text { ity \& } \\
\text { earnings } \\
\text { performa } \\
\text { nce, } \\
\text { turnover, } \\
\text { financial } \\
\text { structure, } \\
\text { size, debt } \\
\text { servicing } \\
\text { capacity, } \\
\text { capital } \\
\text { expendit } \\
\text { ure, } \\
\text { market- } \\
\text { to- book } \\
\text { ratio. }\end{array}$ & $\begin{array}{l}\text { Sample } \\
\text { consisted of top } \\
100 \text { listed } \\
\text { companies on } \\
\text { the Australia } \\
\text { stock exchange } \\
(\text { ASX), using } \\
\text { data collected } \\
\text { from the latest } \\
\text { annual report } \\
\text { and sustainability } \\
\text { report in } 2004 \text {. }\end{array}$ & Nil & $\begin{array}{l}\text { From the study analysis, result indicated a } \\
\text { generally negative relationship between } \\
\text { sustainability disclosure and abnormal } \\
\text { returns. It also showed strong effect of } \\
\text { firm size and industry background. This } \\
\text { imply larger firms will reveal statistically } \\
\text { higher level of sustainability disclosure } \\
\text { than smaller firms. Plausible reason could } \\
\text { be due to the high reliance large firms } \\
\text { place on wider group of internal and } \\
\text { external stakeholders; whose actions can } \\
\text { have different consequence on the firms' } \\
\text { economic performance. This also } \\
\text { signified other determining factors aside } \\
\text { from financial predictors may be } \\
\text { contributing to the level of sustainability } \\
\text { reporting. This study covered only } \\
\text { Australian firms for a single period. }\end{array}$ \\
\hline
\end{tabular}

Table 3: Studies with no significant relationship between sustainability reporting practice and corporate performance

\begin{tabular}{|c|c|c|}
\hline $\mathrm{S} / \mathrm{No}$ & Literature & Main Findings and Comment \\
\hline 1 & $\begin{array}{l}\text { Yilmaz et al. } \\
\text { (2020) } \\
\text { Turkey } \\
\text { (Developing) }\end{array}$ & $\begin{array}{l}\text { Using an event study that measured daily stock returns, the article analyzed effect of } \\
\text { inclusion in and exclusion from the Bursa Istanbul Sustainability Index (BIST SI) on } \\
\text { companies' stocks. However, result showed no strong evidence on both the stock returns } \\
\text { and systematic risk (betas) of the companies. Findings revealed that inclusion reduces the } \\
\text { total risk of the companies by protecting them from stock declines in case of a severe crisis, } \\
\text { and improves their resilience compared to other companies not included. Study suggested } \\
\text { based on findings that investors in the Turkish capital markets does not value corporate } \\
\text { sustainability performance in making their investment decisions probably due to stern belief } \\
\text { of imposing huge costs which reduces profitability on the firm. }\end{array}$ \\
\hline 2 & $\begin{array}{l}\text { Gunarsih and } \\
\text { Ismawati } \\
\text { (2018) } \\
\text { Indonesia } \\
\text { (Developing) }\end{array}$ & $\begin{array}{l}\text { Using a sample of } 60 \text { listed companies in mining, metal and food processing industries on } \\
\text { Indonesia stock exchange IDX for the period between 2014-2017, this study examined the } \\
\text { relationship between sustainability reporting based of GRI and firm performance. Findings } \\
\text { showed two dimensions of SR (economic dimension and social dimension) has an impact } \\
\text { on market value (Tobin's Q) but no impact on book value (ROA). Overall, study found no } \\
\text { relationship between all the dimensions of sustainability reporting with firm performance. } \\
\text { Plausible cause could be because awareness level about the benefits of sustainability } \\
\text { reporting in Indonesia is truncated while reporting is also voluntary. }\end{array}$ \\
\hline 3 & $\begin{array}{l}\text { Ching, Gerab, } \\
\text { and } \quad \text { Toste } \\
(2017) \\
\text { Brazil } \\
\text { (Developing) }\end{array}$ & $\begin{array}{l}\text { For this study, sample comprise of all listed firms on Corporate Sustainability Index in } \\
\text { Brazil over the period } 2008 \text { to } 2014 \text {. The study examined whether sustainability reporting } \\
\text { quality has an effect on corporate financial performance, but findings revealed no clear } \\
\text { consensus. Even though the study found the quality of disclosure to have improved through } \\
\text { the years, there was still no association between accounting and market-based variables and } \\
\text { the reporting quality. This is noticeable since the firm's scores were very low and their } \\
\text { performances deteriorated throughout the years. Possible explanation for the non-consensus } \\
\text { could be because profits from socially responsible conduct failed to compensate for the cost } \\
\text { in a market equilibrium; probably because stakeholders view firms' use costly sustainability } \\
\text { initiative as a legitimization tool to reduce information asymmetry. }\end{array}$ \\
\hline 4 & $\begin{array}{l}\text { Malarvizhi and } \\
\text { Matta (2016) } \\
\text { India } \\
\text { (Developing) }\end{array}$ & $\begin{array}{l}\text { The study investigated the relationship between corporate environmental disclosure and } \\
\text { firm performance for } 85 \text { highly polluting firms listed on the Bombay Stock Exchange } \\
\text { (BSE). Based on a regression analysis, study found no significant relationship. However, } \\
\text { the result revealed a significant correlation with size of companies which signify that large } \\
\text { firms disclose more environmental information in their annual reports and sustainability } \\
\text { reports. Reason for the contrary result could be as a result of the early stage of adoption and } \\
\text { application of GRI by companies operating in the country. }\end{array}$ \\
\hline
\end{tabular}




\begin{tabular}{|c|c|c|}
\hline S/No & Literature & Main Findings and Comment \\
\hline 5 & $\begin{array}{l}\text { Atan, Razali, } \\
\text { Said, and } \\
\text { Zainun (2016). } \\
\text { Denmark and } \\
\text { Malaysia } \\
\text { (Developed and } \\
\text { developing) }\end{array}$ & $\begin{array}{l}\text { The study conducted a comparative analysis on the effect of environmental, social and } \\
\text { governance disclosure on firm performance for Denmark and Malaysia but found no } \\
\text { significant effect. Study revealed there was an absence of legislative pressure in Denmark } \\
\text { which was what drove the level of disclosure higher and more comprehensive than } \\
\text { Malaysia, a country without any specific requisite on ESG. Hence, the study hinged } \\
\text { plausible reason to be due to the temporal lag of disclosure effect on firm's performance, } \\
\text { and the inherent limitation of the environmental value added (EVA) proxy used in the study. } \\
\text { Evidence from this study proved the significance of a country's regulatory background and } \\
\text { the influence it pose on the firm's ESG disclosure level. }\end{array}$ \\
\hline
\end{tabular}

Table 4: Studies with Mixed findings between sustainability reporting practice and corporate performance

\begin{tabular}{|c|c|c|}
\hline S/No & Literature & Main findings and reason for contradictory result \\
\hline 1 & $\begin{array}{l}\text { Akbulut and } \\
\text { Kaya, (2019) } 20 \\
\text { countries }\end{array}$ & $\begin{array}{l}\text { The study provided understandings on the relation of firm performance, firm size, } \\
\text { financial leverage, and sustainability reporting (SR). However, findings showed positive } \\
\text { significant relationship between firm size and SR, but negative significant relationship } \\
\text { was found between financial leverage and SR in the automotive industry. Reason for the } \\
\text { lack of consensus could be due to the study focus which was on only automotive industry } \\
\text { and the proxies used for the variables particularly the inclusion and/or exclusion in the } \\
\text { GRI database. }\end{array}$ \\
\hline 2 & $\begin{array}{l}\text { Kim and Oh } \\
(2019) \text { India } \\
\text { (Developing) }\end{array}$ & $\begin{array}{l}\text { This study explored the relationship between corporate social responsibility (CSR) and } \\
\text { financial performance of Indian firms in the context of both business group firms and } \\
\text { stand-alone firms. Findings revealed CSR disclosure score had a U-shaped relationship } \\
\text { with Tobin's Q. Empirically, the result also revealed that an improvement in CSR actions } \\
\text { does not always result in higher firm value but at the very least, it should exceed a certain } \\
\text { level of CSR to have a positive effect on the value of firms. Furthermore, study showed } \\
\text { that at lower level, a negative relationship between CSR and Tobin's Q weakens in group } \\
\text { affiliate firms but the complement effect of business group disappeared at higher level, } \\
\text { weakening the positive relationship between CSR and Tobin's Q. Hence, creating room } \\
\text { for capital market to grasp the different impact of business groups on CSR performance. } \\
\text { Overall, findings indicated CSR is only related to long term firm performance and not } \\
\text { short-term performance. Possible reason for the contrary opinion may be due to the unique } \\
\text { the traits of Indian firms on CSR practice. This is because most India firms have their } \\
\text { origins deep rooted in philanthropism in the part of community development. }\end{array}$ \\
\hline 3 & $\begin{array}{l}\text { Sampong et al. } \\
\text { (2018). South } \\
\text { Africa } \\
\text { (Developing) }\end{array}$ & $\begin{array}{l}\text { The study investigated the relationship between the extent of corporate social } \\
\text { responsibility (CSR) disclosure and its component and firm value for South African firms. } \\
\text { Based on the panel data fixed effect model, findings revealed positive but insignificant } \\
\text { relationship between CSR disclosure performance and firm value, negative and } \\
\text { insignificant relationship between environmental disclosure performance and firm value; } \\
\text { and a positive and statistically significant relationship between social disclosure } \\
\text { performance and firm value. Overall, findings suggested CSR disclosure had limited } \\
\text { effect on firm value. Further, findings suggested CSR disclosure may not necessarily } \\
\text { influence firm value despite its numerous benefits. Reasons expunge include firms using } \\
\text { disclosure as a legitimizing tool for the actions and inactions; and that different sectors } \\
\text { actively utilize social responsibility policies, not only for growth in global trends and other } \\
\text { external pressures, but also because it could result in efficiency gains for the firm in terms } \\
\text { of profitability and share value. }\end{array}$ \\
\hline 4 & $\begin{array}{l}\text { Miller, Eden, and } \\
\mathrm{Li} \quad(2018) \text { US } \\
\text { (Developed) }\end{array}$ & $\begin{array}{l}\text { This study investigated relationship between changes in corporate social responsibility } \\
\text { (CSR) reputation and firm performance. Result showed changes in CSR reputation have } \\
\text { predictable, asymmetric, and sizeable impacts on firm performance. Findings revealed } \\
\text { that performance impacts depend on whether the firm's CSR reputation in the prior and } \\
\text { current periods is positive (i.e. if the firm performance exceeds CSR regulations); or } \\
\text { performance will be neutral (if performance meets CSR regulations); and performance } \\
\text { will be negative (if performance fails to comply with CSR regulations). This is an } \\
\text { indication that movements toward compliance with CSR regulations will have no } \\
\text { significant impact, but movements away from CSR compliance with CSR regulations will } \\
\text { affect firm performance especially firm's profitability. }\end{array}$ \\
\hline 5 & $\begin{array}{l}\text { Nor, Bahari, } \\
\text { Adnan, Kamal, } \\
\text { and Ali (2016) } \\
\text { Malaysia } \\
\text { (Developing) }\end{array}$ & $\begin{array}{l}\text { The study investigated the effect of environmental disclosure on financial performance } \\
\text { for top } 100 \text { Malaysian firms. Study employed content analysis of annual report for the } \\
\text { year } 2011 \text { and result showed significant relationship between total environmental } \\
\text { disclosure and profit margin but revealed no significant relationship for total } \\
\text { environmental disclosures and ROA, ROE, and EPS. Possible reason could be due to the }\end{array}$ \\
\hline
\end{tabular}




\begin{tabular}{|c|c|c|}
\hline S/No & Literature & Main findings and reason for contradictory result \\
\hline & & $\begin{array}{l}\text { absence of mandatory regulations and statutory requirements for the companies in } \\
\text { Malaysia to disclose environmental sustainability. Moreover, environmental disclosure in } \\
\text { Malaysia is still at infancy, although improving steadily as most companies are already in } \\
\text { the know of environmental awareness. }\end{array}$ \\
\hline 6 & $\begin{array}{l}\text { Garg (2015) } \\
\text { India } \\
\text { (Developing) }\end{array}$ & $\begin{array}{l}\text { The study examined impact of sustainability reporting on firm performance in India. } \\
\text { Result showed negative impact in the short-run but positive impact in the long-run. } \\
\text { Finding also revealed that sustainability reporting practices of companies improved over } \\
\text { the period of analysis. }\end{array}$ \\
\hline 7 & $\begin{array}{l}\text { Bachoo, Tan, and } \\
\text { Wilson (2013). } \\
\text { Australia } \\
\text { (Developed) }\end{array}$ & $\begin{array}{l}\text { The study investigated the relationship between firm value and quality of Australian listed } \\
\text { firms' sustainability reporting. Based on a proprietary data obtained from specialist } \\
\text { responsible investment research firm, the findings from the study document a significant } \\
\text { negative association between quality of sustainability reporting and the cost of equity } \\
\text { capital for listed firms from 2003-2005, but a significant positive association between } \\
\text { expected future performance and the quality of sustainability reporting. The findings rely } \\
\text { on the proposition that markets value high-quality sustainability reporting, and that it is } \\
\text { the environmental component of sustainability reporting that is most closely related to } \\
\text { firm value. }\end{array}$ \\
\hline 8 & $\begin{array}{l}\text { Faisal, Tower, } \\
\text { and Rusmin } \\
(2012) \text { Global }\end{array}$ & $\begin{array}{l}\text { The paper explored corporate sustainability disclosure practices in a global context. The } \\
\text { empirical results revealed that high profile industries and large size companies disclose } \\
\text { more sustainability information. Findings further revealed that firms with additional } \\
\text { voluntary outside assurance statements provide a higher extent of sustainability disclosure } \\
\text { compared with firms without assurance statements. Nevertheless, the result failed to } \\
\text { provide evidence whether board independence contributes to increase in sustainability } \\
\text { disclosure to reflect the impact of strong board governance. Reason expunge for the } \\
\text { findings are that firms often use sustainability reporting as a legitimization tool to } \\
\text { minimize pressure and criticism from society, and to attract capital and build a more } \\
\text { successful business image. }\end{array}$ \\
\hline 9 & $\begin{array}{l}\text { Mohd Taib and } \\
\text { Ameer (2012) } \\
\text { UK and US } \\
\text { (Developed) }\end{array}$ & $\begin{array}{l}\text { This study examined the relationship between corporate sustainability practices and } \\
\text { financial performance using a cross-sectional sample of UK and US listed companies. } \\
\text { Study result showed that extent of disclosure by UK companies supersedes that of US } \\
\text { companies. This is indicative of the significant difference between the financial } \\
\text { performance of UK and US firms in terms of sales growth but no significant difference in } \\
\text { their leverage, ROA and ROE. Meanwhile, their diversity index was found to have } \\
\text { significant positive impact on financial performance, but same was absent for the business } \\
\text { ethics, community and environmental index. }\end{array}$ \\
\hline
\end{tabular}

\section{CONCLUSION}

Corporations that are currently issuing sustainability reports had increased over time and series of empirical studies had been conducted to examine the relationship between sustainability reporting practice and corporate performance. Moreover, commonly adopted theories include stakeholder theory, legitimacy theory, institutional theory and agency theory. Our review study analyzed 35 literatures that have examined this linkage and we came to a conclusive evidence that it pays for firms to adopt sustainable business practices because it has several benefits such as enhancing firm reputation, providing firms access to capital and new markets, assisting firms to gain competitive advantage, etc. Our conclusion is in no denial of the fact that corporations will have to bear some huge costs, but it will only be in the short term. Subsequent to which benefits of sustainability practice will outweigh the cost of investment in the longer period. Although, there is still lack of consensus as findings show contradictory evidences ranging from positive, to negative, to statistically insignificant or mixed results. Several reasons were assumed for the variation. For instance, argument that shareholders view such investment as cost object which stresses profitability; investors does not value the disclosure; or that firms uses disclosure as legitimization tool for prestige; proxies used as measurement tool; country/region covered; weak regulations etc. Based on our outcome, future studies may conduct systematic review to disaggregate the approaches, so as to examine the different dimensions of sustainability practice and provide more concise and clear result. Also, future research may want to investigate the linkage by comparing countries from the same region. For instance, countries in African region over longer period to reveal the long-term effect.

\section{REFERENCES}

Abba, M., Said, R. M., Abdullah, A., \& Mahat, F. (2018). The relationship between environment operational performance and environmental disclosure of Nigerian listed companies. Journal of Environmental Accounting and Management, 6(1), 1-15. https://doi.org/10.5890/JEAM.2018.03.001

Aggarwal, P. (2013). Sustainability Reporting and its Impact on Corporate Financial Performance: A Literature Review. Indian Journal of Commerce and Management Studies, 4(3), 51-59. 
Akbulut, D. H., \& Kaya, I. (2019). Sustainability reporting and firm performance. Pressacademia, 9(9), 81-84. https://doi.org/10.17261/pressacademia.2019.1071

Al-Tuwaijri, S. A., Christensen, T. E., \& Hughes, K. E. (2004). The relations among environmental disclosure, environmental performance, and economic performance: A simultaneous equations approach. Accounting, Organizations and Society, 29(56), 447-471. https://doi.org/10.1016/S0361-3682(03)00032-1

Alcaide González, M. Á., De La Poza Plaza, E., \& Guadalajara Olmeda, N. (2020). The impact of corporate social responsibility transparency on the financial performance, brand value, and sustainability level of IT companies. Corporate Social Responsibility and Environmental Management, 27(2), 642-654. https://doi.org/10.1002/csr.1829

Ameer, R., \& Othman, R. (2012). Sustainability practices and corporate financial performance: A study based on the top global corporations. Journal of Business Ethics, 108(1), 61-79. https://doi.org/10.1007/s10551-011-1063-y

Aras, G., Tezcan, N., \& Kutlu Furtuna, O. (2018). Multidimensional comprehensive corporate sustainability performance evaluation model: Evidence from an emerging market banking sector. Journal of Cleaner Production, 185, 600-609. https://doi.org/10.1016/j.jclepro.2018.01.175

Atan, R., Razali, F. A., Said, J., \& Zainun, S. (2016). Environmental, social and governance (esg) disclosure and its effect on firm's performance: A comparative study. International Journal of Economics and Management, 10(Specialissue2), 355-375. Retrieved from http://www.econ.upm.edu.my/ijem

Bachoo, K., Tan, R., \& Wilson, M. (2013). Firm Value and the Quality of Sustainability Reporting in Australia. Australian Accounting Review, 23(1), 67-87. https://doi.org/10.1111/j.1835-2561.2012.00187.x

Borges Junior, D. M. (2019). Sustainability report and performance of Brazilian public companies. Revista Catarinense Da Ciência Contábil, 18(2014), 1-12. https://doi.org/10.16930/2237-766220192779

Brown, N., \& Deegan, C. (1998). The public disclosure of environmental performance information - A dual test of media agenda setting theory and legitimacy theory. Accounting and Business Research, 29(1), 21-41. https://doi.org/10.1080/00014788.1998.9729564

Buallay, A. (2019). Is sustainability reporting (ESG) associated with performance? Evidence from the European banking sector. Management of Environmental Quality: An International Journal, 30(1), 98-115. https://doi.org/10.1108/MEQ-12-2017-0149

Ching, H. Y., Gerab, F., \& Toste, T. H. (2017). The Quality of Sustainability Reports and Corporate Financial Performance: Evidence From Brazilian Listed Companies. SAGE Open, 7(2). https://doi.org/10.1177/2158244017712027

Chithambo, L., Tingbani, I., Agyapong, G. A., Gyapong, E., \& Damoah, I. S. (2020). Corporate voluntary greenhouse gas reporting: Stakeholder pressure and the mediating role of the chief executive officer. Business Strategy and the Environment, 29(4), 16661683. https://doi.org/10.1002/bse. 2460

Clarkson, M. E. (1995). a Stakeholder Framework for Analyzing and Evaluating Corporate Social Performance. Academy of Management Review, 20(1), 92-117. https://doi.org/10.5465/amr.1995.9503271994

Cornett, M. M., Erhemjamts, O., \& Tehranian, H. (2016). Greed or good deeds: An examination of the relation between corporate social responsibility and the financial performance of U.S. commercial banks around the financial crisis. Journal of Banking and Finance, 70, 137-159. https://doi.org/10.1016/j.jbankfin.2016.04.024

Deegan, C. (2002). Introduction: The legitimising effect of social and environmental disclosures - a theoretical foundation. Accounting, Auditing \& Accountability Journal, 15(3), 282-311. https://doi.org/10.1108/09513570210435852

Delai, I., \& Takahashi, S. (2013). Corporate sustainability in emerging markets: Insights from the practices reported by the Brazilian retailers. Journal of Cleaner Production, 47, 211-221. https://doi.org/10.1016/j.jclepro.2012.12.029

Detre, J. D., \& Gunderson, M. A. (2011). The triple bottom line: What is the impact on the returns to agribusiness? International Food and Agribusiness Management Review, 14(4), 165-178.

Dinçer, Ö., \& Altınay, A. (2020). The Effect of Declarations of Sustainability Reports on Financial Performances of Banks in Turkey. (January), 1-15. https://doi.org/10.20944/preprints202001.0145.v1

Donaldson, T., \& Preston, L. E. (1995). Theory the Stakeholder of the Concepts, Evidence, Corporation : and Implications. Academy of Management Review, 20(1), 65-91. https://doi.org/10.2307/258887

Elkington, J. (1999). Triple bottom-line reporting: Looking for balance. AUSTRALIAN CPA, 69, 18-21.

Emeka-Nwokeji, N. A., \& Okeke, P. C. (2019). Evaluating Corporate Environmental Disclosures and Performance of Quoted NonFinancial Firms in Nigeria. Journal of Accounting, Business and Social Sciences, 1(2), 119-132.

Faisal, F., Tower, G., \& Rusmin, R. (2012). Legitimising Corporate Sustainability Reporting Throughout the World. Australasian Accounting Business \& Finance Journal, 6(2), 19-34.

Fatemi, A., Glaum, M., \& Kaiser, S. (2018). ESG performance and firm value: The moderating role of disclosure. Global Finance Journal, 38, 45-64. https://doi.org/10.1016/j.gfj.2017.03.001

Freeman, R. E. (1984). Stakeholder management: framework and philosophy. Pitman, Mansfield, MA.

Freeman, R. E., \& David, L. R. (1983). Stockholders and Stakeholders: A New Perspective on Corporate Governance. California Management Review, 25(3), 88-106. https://doi.org/10.2307/41165018

Freeman, R. E., Harrison, J. S., Wicks, A. C., Parmar, B. L., \& De Colle, S. (2010). Stakeholder theory: The state of the art. Cambridge University Press.

Furlan Alves, M. B., Lopes de Sousa Jabbour, A. B., \& Barberio Mariano, E. (2019). How can we solve the puzzle of strategic climate management and appreciate its long-term effects? Journal of Organizational Change Management, 32(7), 687-708. https://doi.org/10.1108/JOCM-01-2018-0013

Garcia, A. S., Mendes-Da-Silva, W., \& Orsato, R. (2017). Sensitive industries produce better ESG performance: Evidence from emerging markets. Journal of Cleaner Production, 150, 135-147. https://doi.org/10.1016/j.jclepro.2017.02.180

Garg, P. (2015). Impact of Sustainability Reporting on Firm Performance of Companies in India. International Journal of Marketing and Business Communication, 4(3). https://doi.org/10.21863/ijmbc/2015.4.3.018

Global Reporting Initiative. (2002). The GRI Standards: the global standards for sustainability reporting. Retrieved from www.globalreporting.org

Global Reporting Initiative. Gri 101: foundation 2016 101. , GRI101 GRI Standards § (2016).

Gunarsih, T., \& Ismawati, Y. (2018). Sustainability Report and Firm Performance: Study in Mining and Metal and Food Processing Industry Indonesia Stock Exchange 2014-2017. Journal of Governance and Integrity, 2(1), 9-26.

Henriques, A., \& Richardson, J. (2004). The triple bottom line, does it all add up?: assessing the sustainability of ... In The Triple 
Bottom Line (p. 186). Retrieved from http://books.google.com/books?id=JliHcXcLSHAC\&pgis=1

Hoejmose, S. U., Roehrich, J. K., \& Grosvold, J. (2014). Is doing more doing better? The relationship between responsible supply chain management and corporate reputation. Industrial Marketing Management, 43(1), $77-90$. https://doi.org/10.1016/j.indmarman.2013.10.002

Ioannou, I., \& Serafeim, G. (2017). The Consequences of Mandatory Corporate Sustainability Reporting. Retrieved from https://pdfs.semanticscholar.org/f44a/77e9799017edb8a2a90e00b2c2ba742ea2f2.pdf

Jan, A., Marimuthu, M., bin Mohd, M. P., \& Isa, M. (2019). The nexus of sustainability practices and financial performance: From the perspective of Islamic banking. Journal of Cleaner Production, 228, 703-717. https://doi.org/10.1016/j.jclepro.2019.04.208

Jones, S., Frost, G., Loftus, J., \& Van Der Laan, S. (2007). An Empirical examination of the market returns and financial performance of entities engaged in sustainability reporting. Australian Accounting Review, 17(41), 78-87. https://doi.org/10.1111/j.18352561.2007.tb00456.x

Joshi, S., Pandey, V., \& Ross, R. B. (2017). Asymmetry in Stock Market Reactions to Changes in Membership of the Dow Jones Sustainability Index. The Journal of Business Inquiry, 16(1), 12-35. Retrieved from http:www.uvu.edu/woodbury/jbi/articles/

Kim, W. S., \& Oh, S. (2019). Corporate social responsibility, business groups and financial performance: a study of listed Indian firms. Economic Research-Ekonomska Istrazivanja, 32(1), 1777-1793. https://doi.org/10.1080/1331677X.2019.1637764

KPMG. (2017). The road ahead: KPMG International Survey of Corporate Responsibility Reporting. In KPMG Reporting. Retrieved from www.kpmg.com/crreporting

Kwaghfan, A. (2015). Impact of Sustainability Reporting on Corporate Performance of Selected Quoted Companies in Nigeria.

Lin, C. H., Yang, H. L., \& Liou, D. Y. (2009). The impact of corporate social responsibility on financial performance: Evidence from business in Taiwan. Technology in Society, 31(1), 56-63. https://doi.org/10.1016/j.techsoc.2008.10.004

Loh, L., Thomas, T., \& Wang, Y. (2017). Sustainability reporting and firm value: Evidence from Singapore-listed companies. Sustainability (Switzerland), 9(11), 1-12. https://doi.org/10.3390/su9112112

López, M. V., Garcia, A., \& Rodriguez, L. (2007). Sustainable development and corporate performance: A study based on the Dow Jones sustainability index. Journal of Business Ethics, 75(3), 285-300. https://doi.org/10.1007/s10551-006-9253-8

Malarvizhi, P., \& Matta, R. (2016). Link between Corporate Environmental Disclosure and Firm Performance : Perception or Reality? Review of Integrative Business and Economics Research, 5(3), 1-34. Retrieved from https://search.proquest.com/agricenvironm/docview/1814289754/fulltextPDF/43593F5413684851PQ/8?accountid=48290

Maletič, M., Maletič, D., \& Gomišček, B. (2018). The role of contingency factors on the relationship between sustainability practices and organizational performance. Journal of Cleaner Production, 171, 423-433. https://doi.org/10.1016/j.jclepro.2017.09.172

Margolis, J. D., \& Walsh, J. P. (2003). Misery Loves Companies: Rethinking Social Initiatives by Business. Administrative Science Quarterly, 48(2). https://doi.org/10.2307/3556659

Miller, S. R., Eden, L., \& Li, D. (2018). CSR Reputation and Firm Performance: A Dynamic Approach. Journal of Business Ethics, $O(0), 0$. https://doi.org/10.1007/s10551-018-4057-1

Mohd Taib, E., \& Ameer, R. (2012). Relationship between Corporate Sustainability Practices and Financial Performance: Evidence from the GRI Reporting Companies. SSRN Electronic Journal, 1-27. https://doi.org/10.2139/ssrn.2152124

Muhmad, S. N., \& Muhamad, R. (2020). Sustainable business practices and financial performance during pre- and post-SDG adoption periods: a systematic review. Journal of Sustainable Finance and Investment. https://doi.org/10.1080/20430795.2020.1727724

N. Burhan, A. H., \& Rahmanti, W. (2012). the Impact of Sustainability Reporting on Company Performance. Journal of Economics, Business, and Accountancy | Ventura, 15(2), 257. https://doi.org/10.14414/jebav.v15i2.79

Ng, A. C., \& Rezaee, Z. (2012). Sustainability Disclosures and Cost of Capital. SSRN Electronic Journal, (852). https://doi.org/10.2139/ssrn.2038654

Nor, N. M., Bahari, N. A. S., Adnan, N. A., Kamal, S. M. Q. A. S., \& Ali, I. M. (2016). The Effects of Environmental Disclosure on Financial Performance in Malaysia. Procedia Economics and Finance, 35(October 2015), 117-126. https://doi.org/10.1016/s2212-5671(16)00016-2

NSE, N. S. E. (2018). Circular on Sustainability Disclosure Guidelines. Retrieved from Circular NSE/RD/LRD/CIR1/18/12/19 website: http://www.nse.com.ng/mediacenter/pressreleases/Pages/NSE-Announces-its-Sustainability-Disclosure-Guidelines.aspx

Patten, D. M. (1991). Exposure, legitimacy, and social disclosure. Journal of Accounting and Public Policy, 10(4), $297-308$. https://doi.org/10.1016/0278-4254(91)90003-3

Rajesh, R. (2020). Exploring the sustainability performances of firms using environmental, social, and governance scores. Journal of Cleaner Production, 247, 119600. https://doi.org/10.1016/j.jclepro.2019.119600

Rajesh, R., \& Rajendran, C. (2020). Relating Environmental, Social, and Governance scores and sustainability performances of firms: An empirical analysis. Business Strategy and the Environment, 29(3), 1247-1267. https://doi.org/10.1002/bse.2429

Reddy, K., \& Gordon, L. W. (2010). The effect of sustainability reporting on financial performance: An empirical study using listed companies. Journal of Asia Entrepreneurship and Sustainability, 6(2), 19-42.

Rezaee, Z. (2016). Business sustainability research: A theoretical and integrated perspective. Journal of Accounting Literature, 36, 4864. https://doi.org/10.1016/j.acclit.2016.05.003

Sampong, F., Song, N., Boahene, K. O., \& Wadie, K. A. (2018). Disclosure of CSR performance and firm value: New evidence from South Africa on the basis of the GRI guidelines for sustainability disclosure. Sustainability (Switzerland), 10(12). https://doi.org/10.3390/su10124518

Schmidt, C. G., Foerstl, K., \& Schaltenbrand, B. (2017). The Supply Chain Position Paradox: Green Practices and Firm Performance. Journal of Supply Chain Management, 53(1), 3-25. https://doi.org/10.1111/jscm.12113

Shad, M. K., Lai, F. W., Fatt, C. L., Klemeš, J. J., \& Bokhari, A. (2019). Integrating sustainability reporting into enterprise risk management and its relationship with business performance: A conceptual framework. Journal of Cleaner Production, 208, 415-425. https://doi.org/10.1016/j.jclepro.2018.10.120

Suchman, M. C. (1995). Managing Legitimacy: Strategic and Institutional Approaches. Academy of Management Review, 20(3), 571610. https://doi.org/doi.org/10.5465/amr.1995.9508080331

Tarmuji, I., Maelah, R., \& Tarmuji, N. H. (2016). The Impact of Environmental, Social and Governance Practices (ESG) on Economic Performance: Evidence from ESG Score. International Journal of Trade, Economics and Finance, 7(3), 67-74. https://doi.org/10.18178/ijtef.2016.7.3.501

Wei, J. (2020). Environmental, Social, and Governance Proposals and Shareholder Activism. The Journal of Portfolio Management, 
46(3), 49-64. https://doi.org/10.3905/jpm.2020.46.3.049

World Commission on Environment and Development. (1987). The Brundtland Report: In Our Common Future'. https://doi.org/10.1080/07488008808408783

Yilmaz, M. K., Aksoy, M., \& Tatoglu, E. (2020). Does the stock market value inclusion in a sustainability index? Evidence from Borsa Istanbul. Sustainability (Switzerland), 12(2). https://doi.org/10.3390/su12020483

Zhao, C., Guo, Y., Yuan, J., Wu, M., Li, D., Zhou, Y., \& Kang, J. (2018). ESG and corporate financial performance: Empirical evidence from China's listed power generation companies. Sustainability (Switzerland), 10(8), 1-18. https://doi.org/10.3390/su10082607

\section{ACKNOWLEDGEMENT}

The authors would like to thank the editor and anonymous referees for their insightful comments on the earlier draft of this paper. Gratitude also to my supervisor for her comments and constructive guidance towards the success of this paper.

\section{DECLARATION CONFLICT OF INTEREST}

We declare not having any potential competing interests or personal relationships that can influence the work reported in this study.

\section{AUTHORS' BIOGRAPHY}

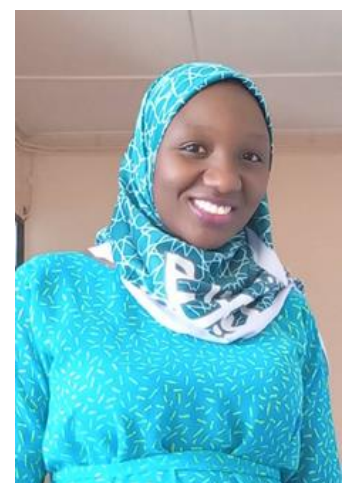

Nusirat Ojuolape Gold is a current PhD. Student of Accounting at the School of Management, Universiti Sains Malaysia under the tutelage of Professor Fauziyah Md Taib.

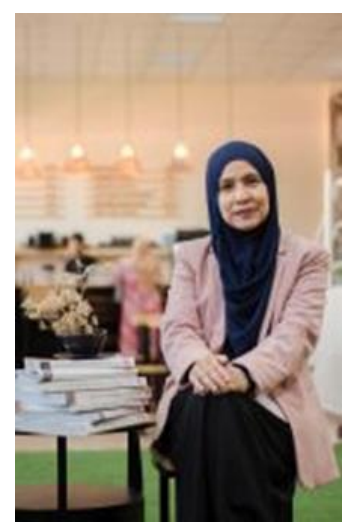

Fauziyah Md Taib is a professor of Accounting at the School of Management, Universiti Sains Malaysia. 